JULIO CESAR NATHER JUNIOR

\title{
DETECÇÃO AUTOMÁTICA DE LESÕES NOVAS EM EXAMES CONSECUTIVOS DE PACIENTES COM ESCLEROSE MÚLTIPLA
}

Dissertação apresentada à Faculdade de Medicina de Ribeirão Preto da Universidade de São Paulo para obtenção do título de Mestre em Ciências.

Área de Concentração:

Mestrado Profissional em Ciências das Imagens e Física Médica

Orientador:

Prof. Dr. Carlos Ernesto Garrido Salmon

Ribeirão Preto

2019 


\section{AUTORIZO A REPRODUÇÃO E DIVULGAÇÃO TOTAL DESTE TRABALHO, POR QUALQUER MEIO CONVENCIONAL OU ELETRÔNICO, PARA FINS DE ESTUDO E PESQUISA, DESDE QUE CITADA A FONTE.}

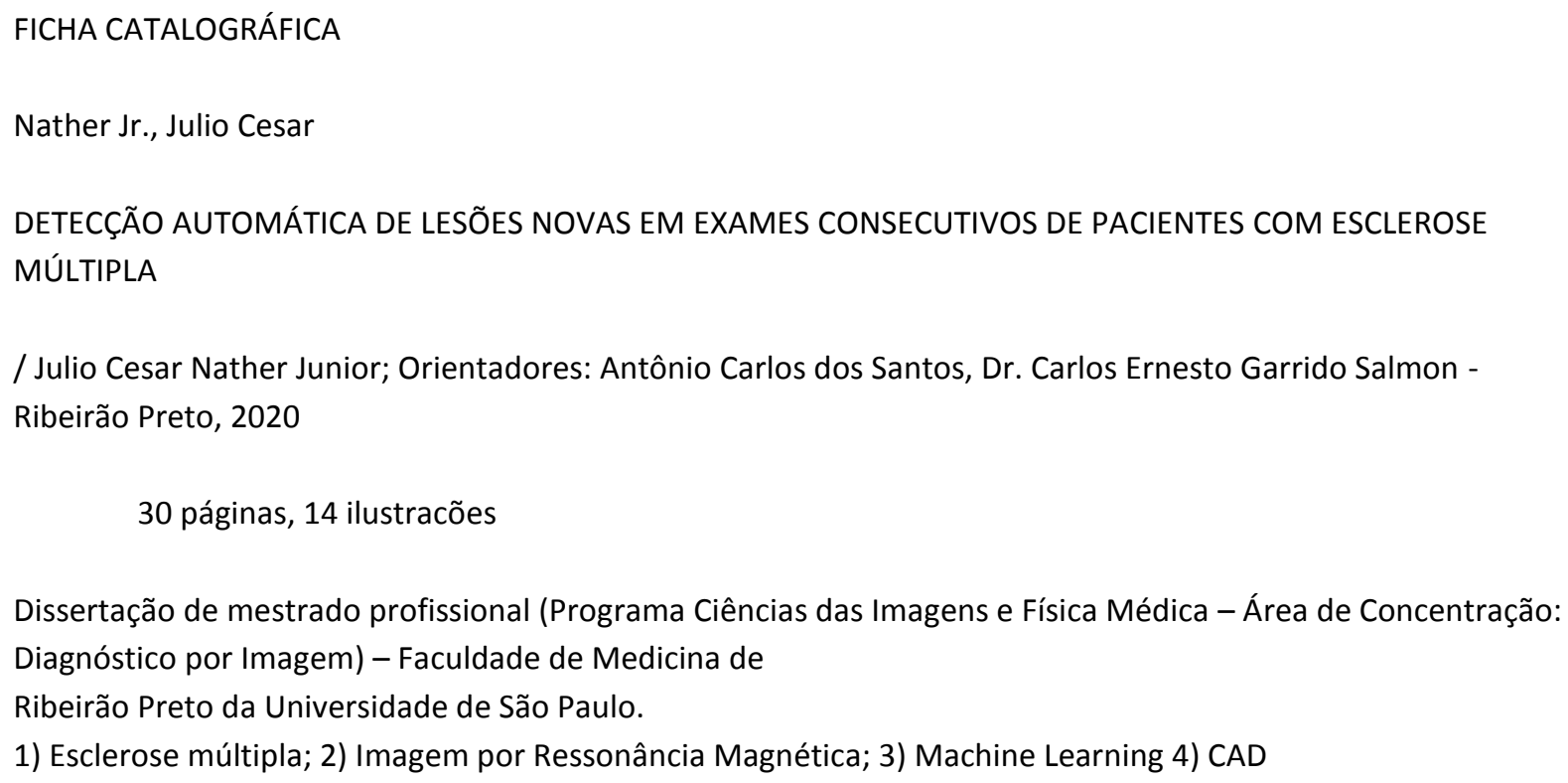


FOLHA DE APROVAÇÃO

DETECÇÃO AUTOMÁTICA DE LESÕES NOVAS EM EXAMES CONSECUTIVOS DE PACIENTES COM ESCLEROSE MÚLTIPLA

Dissertação apresentada à Faculdade de Medicina de Ribeirão Preto da Universidade de São Paulo para obtenção do título de mestre profissionalizante.

Áreas de concentração: Diagnóstico por Imagem e Computação

Aprovado em ___

Banca examinadora:

Prof. Dr

Instituicão:

Assinatura:

Prof. Dr Instituicão:

Assinatura:

Prof. Dr Instituicão:

Assinatura: 


\section{AGRADECIMENTOS}

Aos meus pais, Julio e Sandra, pelo apoio incondicional, conselhos e ensinamentos, sem os quais nada seria possível.

À minha querida esposa, Carla, pelo carinho, incentivo e compreensão nos momentos difíceis.

Aos professores Antônio Carlos dos Santos e Carlos Ernesto Garrido Salmon pela ajuda, confiança em meu trabalho e valiosa contribuição em meu conhecimento científico. 


\section{RESUMO}

\section{Julio Cesar Nather Junior. Deteç̧ão automática de lesões novas em exames consecutivos de pacientes com esclerose múltipla. Dissertação de mestrado profissional. Faculdade de Medicina de Ribeirão Preto, Universidade de São Paulo.}

Embasamento: A Esclerose Múltipla (EM) é uma doença desmielinizante inflamatória restrita ao sistema nervoso central, caracterizada pela ocorrência de inflamação focal com distribuição perivenular. Os critérios diagnósticos da doença foram revisados em 2017, sendo conhecidos como critérios de McDonald e se baseiam nos princípios de dissociação temporal e espacial das lesões. Apesar de ser conhecido que a doença acomete virtualmente qualquer porção do SNC, sendo predominante na substância branca (SB), porém, não exclusiva, envolvendo também córtex e substância cinzenta (SC) profunda. A identificação de recorrência da doença se baseia na identificação de focos macroscópicos de desmielinização, conhecidos como placas. In vivo, o diagnóstico das placas depende da sua identificação em exames de imagem ressonância magnética (MRI). Os pacientes com EM são submetidos a exames de MRI do encéfalo com o intuito de se identificar lesões novas ou aumento do volume total de lesões. A lesão aparece no exame de MRI como uma imagem de hiperintensidade de sinal em sequências T2, que pode ser isolada, medindo mais que 3 a $5 \mathrm{~mm}$, ovaladas, com realce após administração de contraste paramagnético intravenoso na fase aguda. Cronicamente as placas se tornam menos delimitáveis, coalescentes, formando aglomerados dismórficos com predomínio no corpo caloso e região de SB periventricular. A detecção de novas lesões entre dois exames de MRI é critério para a definição de estabilidade ou progressão de doença, bem como o sucesso ou fracasso terapêutico. Em pacientes com alta carga lesional, a detecção de uma nova e pequena lesão não é tarefa trivial para o médico radiologista. Objetivo: Para aumentar a sensibilidade e especificidade do exame é a proposta deste trabalho o desenvolvimento de um algoritmo computacional para volumetria de lesões e comparação entre diferentes tempos de estudo a fim de identificar lesões novas. Casuística e Método: Foram analisadas retrospectivamente as imagens de 99 pacientes com diagnóstico confirmado de Esclerose Múltipla e acompanhados clinicamente no HCFMRP-USP no período de 2013 a 2020 com imagens adquiridas um equipamento de 3T (Philips, Achieva). A imagem volumétrica FLAIR de dois exames consecutivos foram segmentadas e corregistradas. Uma ferramenta para detecção de novas lesões foi implementada, comparando exames prévios e recentes por software de detecção automática de lesões. Resultados: Foi observada uma sensibilidade de $95 \%$ e especificidade de $31 \%$. Somente houve um falso negativo entre os 99 exames avaliados. Conclusão: o algoritmo desenvolvido foi considerado efetivo, com alta sensibilidade e funcionando de forma totalmente automática. A prática diária demonstrou que houve um auxílio real na rotina de avaliação dos exames, aumentando a rapidez e confiabilidade da avaliação. A baixa especificidade não compromete a eficiência, sendo os falsos positivos rapidamente descartados pelo médico radiologista.

Palavras-chave: esclerose múltipla, inteligência artificial, detecção automática de lesões, segmentação automática, volumetria. 


\begin{abstract}
Julio Cesar Nather Junior. Automatic detection of new injuries in consecutive examinations of patients with multiple sclerosis. Master's thesis. Ribeirão Preto School of Medicine, University of São Paulo.
\end{abstract}

Background: Multiple Sclerosis (MS) is an inflammatory demyelinating disease restricted to the central nervous system, characterized by the occurrence of focal inflammation with perivenular distribution. The diagnostic criteria for the disease were revised in 2017, known as McDonald's criteria and are based on the principles of temporal and spatial dissociation of injuries. Although it is known that the disease affects virtually any portion of the CNS, being predominant in white matter (SB), however, not exclusive, also involving cortex and deep gray matter (SC). The identification of disease recurrence is based on the identification of macroscopic foci of demyelination, known as plaques. In vivo, the diagnosis of plaques depends on their identification in magnetic resonance imaging (MRI) exams. Patients with MS are submitted to brain MRI exams in order to identify new lesions or increase the total lesion volume. The lesion appears on the MRI exam as an image of signal hyperintensity in T2 sequences, which can be isolated, measuring more than 3 to $5 \mathrm{~mm}$, oval, with enhancement after intravenous paramagnetic contrast in the acute phase. Chronically, the plaques become less delimitable, coalescent, forming dysmorphic clusters with predominance in the corpus callosum and periventricular SB region. The detection of new lesions between two MRI exams is a criterion for defining disease stability or progression, as well as therapeutic success or failure. In patients with a high lesion load, the detection of a new and small lesion is not a trivial task for the radiologist. Objective: To increase the sensitivity and specificity of the exam, the purpose of this work is to develop a computational algorithm for lesion volumetry and to compare different study times in order to identify new lesions. Casuistic and Method: The images of 99 patients with confirmed diagnosis of Multiple Sclerosis were retrospectively analyzed and followed up clinically at HCFMRP-USP in the period from 2013 to 2020 with images acquired from a 3T equipment (Philips, Achieva). The volumetric FLAIR image of two consecutive exams was segmented and registered. A tool for detecting new injuries was implemented, comparing previous and recent exams using automatic injury detection software. Results: A sensitivity of $95 \%$ and specificity of $31 \%$ were observed. There was only one false negative among the 99 tests evaluated. Conclusion: the developed algorithm was considered effective, with high sensitivity and working in a fully automatic way. Daily practice demonstrated that there was a real help in the routine of exam assessment, increasing the speed and reliability of the assessment. Low specificity does not compromise efficiency, and false positives are quickly discarded by the radiologist.

Keywords: multiple sclerosis, artificial intelligence, automatic lesion detection, automatic segmentation, volumetry. 
SUMÁRIO

\begin{tabular}{|l|l|l|l|}
\hline 01 & & & Capa \\
\hline 04 & & & Agradecimentos \\
\hline 05 & & & Resumo \\
\hline 06 & & & Abstract \\
\hline 08 & & & Introdução \\
\hline 12 & & & Objetivo \\
\hline 13 & & & Casuística e Método \\
\hline 18 & & & Resultados \\
\hline 25 & & & Discussão \\
\hline 31 & & & Conclusão \\
\hline 32 & & & Bibliografia \\
\hline
\end{tabular}




\section{Introdução}

A Esclerose Múltipla (EM) é uma doença desmielinizante inflamatória restrita ao sistema nervoso central. Sua forma clínica mais comum é a alternância entre surtos clínicos, caracterizados por déficits focais com origem na medula espinhal, nervo óptico e parênquima encefálico. A multiplicidade de locais afetados é uma das marcas da doença, caracterizando a dissociação espacial das lesões. A forma clínica mais comum é caracterizada pela alternância de surtos com períodos de remissão, com recuperação total ou parcial do déficit, caracterizando um envolvimento multifásico e uma distribuição temporal, forma denominada recorrente e remissão (RR). Após um período variável de alguns anos, é comum a doença iniciar uma fase crônica de progressão, com ou sem alguns surtos, conhecida como fase secundariamente progressiva (SP). Cerca de $10 \%$ dos casos evoluem com progressão ininterrupta dos sintomas, sendo denominada forma primariamente progressiva (PP). A EM é uma doença com baixa mortalidade, porém, com longa evolução e alta morbidade decorrente do acúmulo progressivo de déficits neurológicos relacionados com resquícios de recuperação incompleta dos surtos sucessivos. As manifestações neurológicas são extremamente variadas, determinadas pela extensão e pelo local dos focos de desmielinização inflamatória, comumente denominadas placas. Estas lesões ocorrem em qualquer local da substância branca do SNC, porém, sua manifestação clínica é mais comumente percebida quando acometem determinados locais do sistema nervoso central denominados eloquentes, relacionados com funções neurológicas motoras, sensitivas ou visuais. As lesões ocorrem em tamanho muito variado, sendo as lesões macroscópicas, resultando em sinais, sintomas e achados radiológicos que podem ser reconhecidas como características da esclerose múltipla (1). A esclerose múltipla tem incidência diferente nos diversos locais do mundo, sendo mais prevalente em países acima ou abaixo dos trópicos e menos prevalente nas regiões tropicais. No Brasil estima-se 10 para cada 100.000 habitantes. A EM é caracterizada clinicamente por diversos sinais e sintomas dependendo das regiões onde ocorre a desmielinização. No Sistema Nervoso Central, estes pacientes apresentam lesões na forma de placas endurecidas, por isso o nome esclerose (2). Por ser uma 
doença crônica, o tratamento consiste no uso por tempo indeterminado de medicamentos, dependendo da forma de apresentação da doença e da evolução. Devido ao espectro de manifestações clínicas, o paciente geralmente demanda uma equipe multidisciplinar para o seu tratamento.

Os pacientes com EM são submetidos a exames de imagem do encéfalo periódicos. A Ressonância Magnética (RM) é a principal técnica usada tanto para o diagnóstico como para seguimento dos pacientes. A detecção de novas lesões entre dois exames de RM pelo médico radiologista é fundamental devido à importância para guiar o tratamento e para o prognóstico (3). Novas lesões em pacientes usando medicações específicas podem ser um indicativo de ineficácia do tratamento.

A detecção de lesões novas exige acentuada atenção por parte do médico radiologista, principalmente em pacientes que possuem muitas lesões pré-existentes (Figura 1). Deve ser destacado que estas lesões têm sua origem na microestrutura cerebral, assim seu tamanho pode ser muito pequeno dificultando sua identificação. Esta tarefa se torna ainda mais difícil quando se evidenciam outras diversas alterações no exame e pelo tempo cada vez mais reduzido para a elaboração dos laudos frente ao crescente número de exames feitos nos serviços de radiologia. 


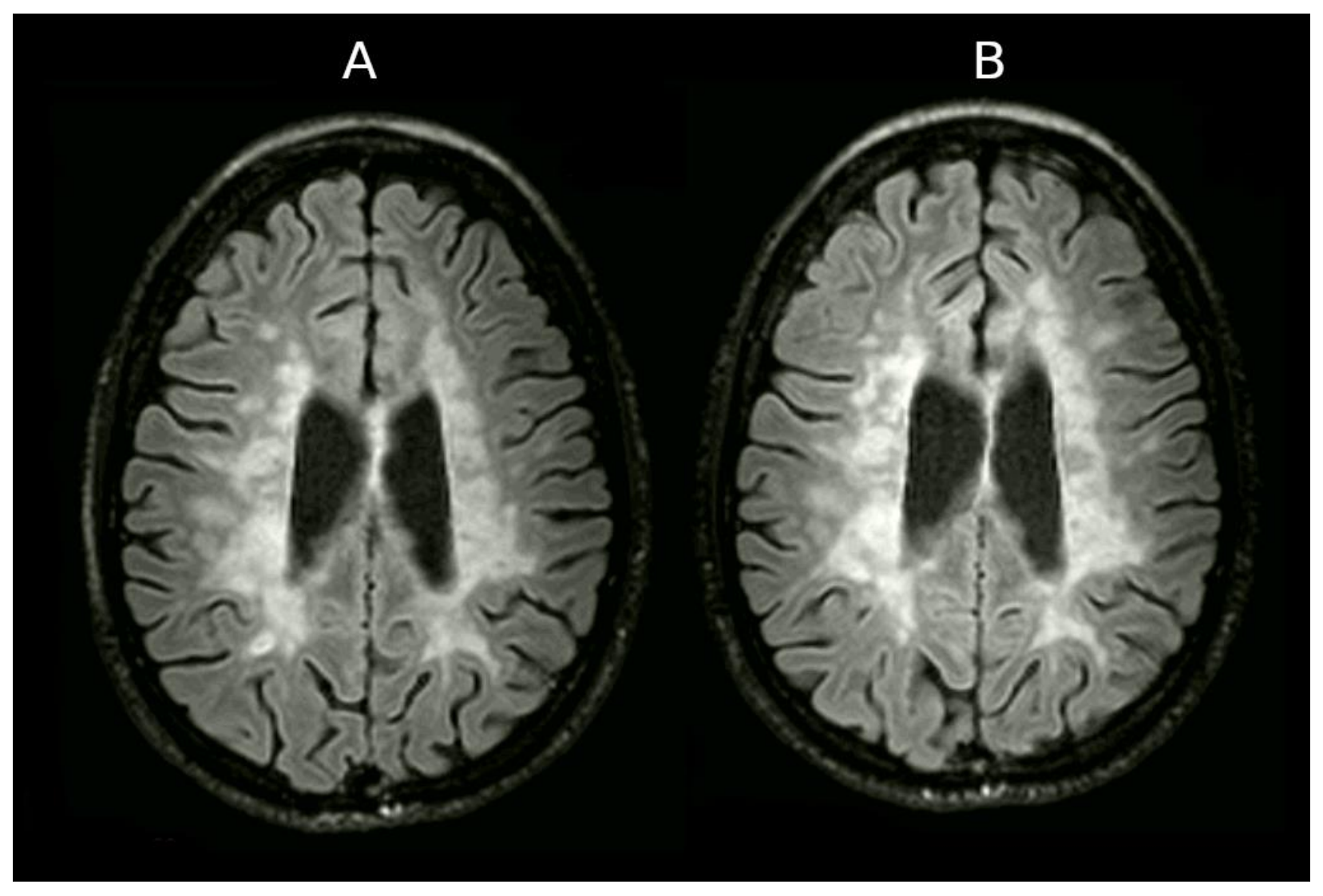

Figura 1: Exemplo de paciente com EM com muitas pequenas lesões em dois exames consecutivos de RM, tornando muito difícil a tarefa de identificação de novas lesões. a) exame prévio, b) exame atual.

Nos últimos anos o uso das técnicas de aprendizado de máquina tem ganhado um grande espaço na área de imagens médicas (4). Estas técnicas procuram por padrões para a realização de problemas complexos, porém que sua solução possa ser "aprendida" por uma máquina a partir de exemplos de solução previamente conhecidos. Desta forma, a identificação de lesões de EM em imagens por RM é uma tarefa que pode se beneficiar deste recurso. Encontramos na literatura diversos trabalhos publicados que propõem alternativas para essa tarefa $(5,6)$. Porém, eles apresentam diversos graus de sensibilidade e especificidade. Em um trabalho mais recente (7), foi utilizado métodos de corregistro e fusão de imagens demonstrando alta sensibilidade e especificidade intra e interobservadores, porém este 
método ainda depende da tediosa comparação e procura por novas imagens por parte do radiologista. 


\section{OBJETIVOS}

Nesse panorama propusemos o desenvolvimento e a aplicação de uma ferramenta de auxílio ao médico radiologista para a avaliação de dois exames consecutivos de imagens por RM de pacientes com Esclerose Múltipla visando a detecção automática de novas lesões. Para esse desenvolvimento foram definidos os seguintes objetivos específicos:

- Disponibilização automática dos exames de RM de pacientes com EM adquiridos nas máquinas do HCFMRP-USP.

- Detecção de novas lesões a partir de duas séries FLAIR de um paciente de EM adquiridas em tempos diferentes.

- Incorporação automática do resultado da deteç̧ão nos arquivos do segundo exame do paciente. 


\section{Casuística e métodos}

Foram analisadas retrospectivamente as imagens de 99 pacientes com diagnóstico confirmado de Esclerose Múltipla e acompanhados clinicamente no HCFMRP-USP no período de 2013 a 2020. Entre os pacientes avaliados a idade média foi de 32 anos e 63 pacientes foram do sexo feminino. Os pacientes considerados neste estudo não assinaram o termo de consentimento livre e esclarecido uma vez que se trata de estudo retrospectivo, com análise de exames de imagem e do prontuário, sem nenhum novo procedimento envolvido.

As imagens de RM foram inicialmente adquiridas em um equipamento de 3T (Philips, Achieva) usando uma bobina de cabeça de 8 canais de recepção. Estas imagens estavam armazenadas nos servidores do Centro de Imagens e Física Médica do HCRP. Ao todo 99 exames foram avaliados, sendo que diferentes pacientes possuíam diferentes números de exames.

As figuras 2, 3 e 4 representam fluxogramas da ferramenta aqui elaborada separada por etapas, sendo que cada etapa envolve cada um dos objetivos específicos deste trabalho. Dessa forma, as etapas foram chamadas de Seleção (Figura 2), Processamento (Figura 3) e Visualização (Figura 4). A seguir descrevemos mais detalhadamente cada uma delas. 
Figura 2: o script python checa a cada 5 minutos

\section{SELEÇÃO}

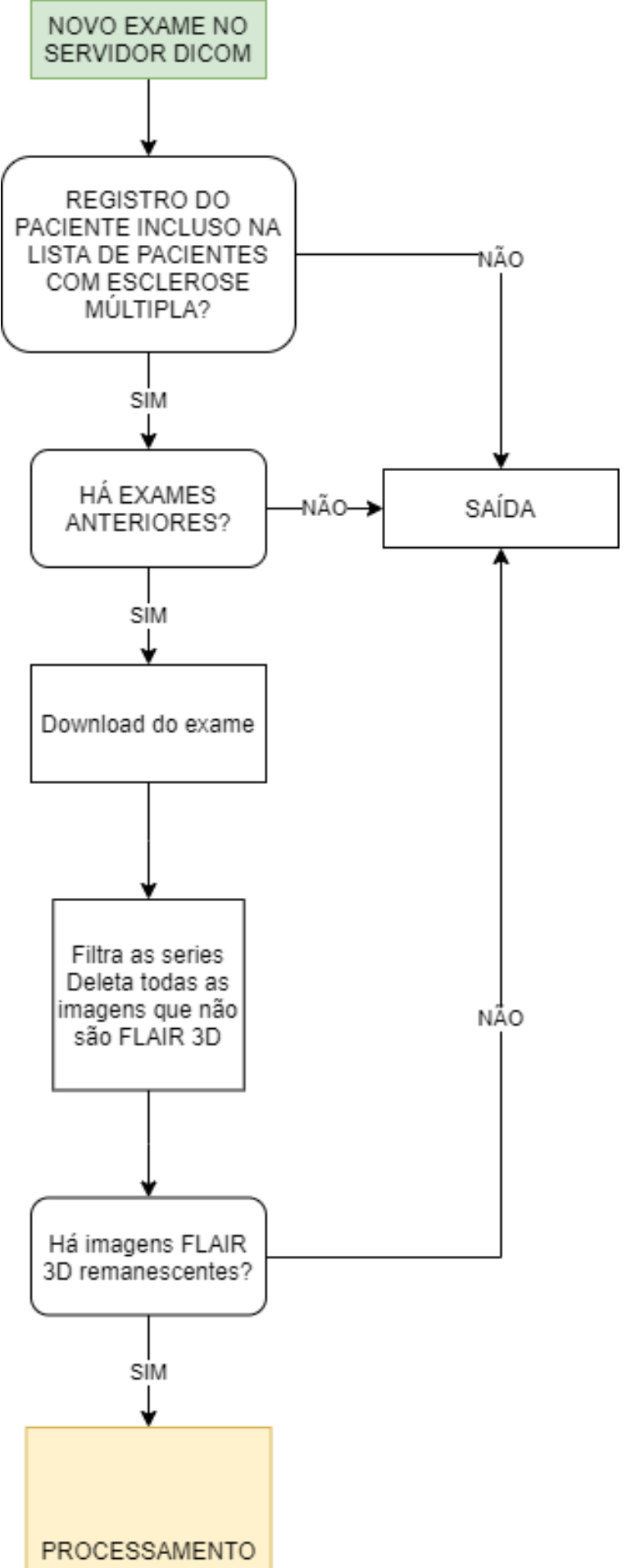

se há exames novos no servidor. Caso positivo, checa se o registro do paciente do exame novo está na lista de paciente em seguimento de esclerose múltipla no nosso banco de dados. Caso positivo, faz o download do exame e realiza a filtragem das imagens baseando-se em diversos critérios presentes nas tags DICOM, excluindo todas as imagens que não preenche estes critérios, a fim de selecionar somente imagens de sequência FLAIR 3D que será usada no processamento. Ao final desta etapa, há uma checagem se houve seleção das imagens, caso positivo o exame é registrado no banco de dados e marcado para processamento. 


\section{PROCESSAMENTO}

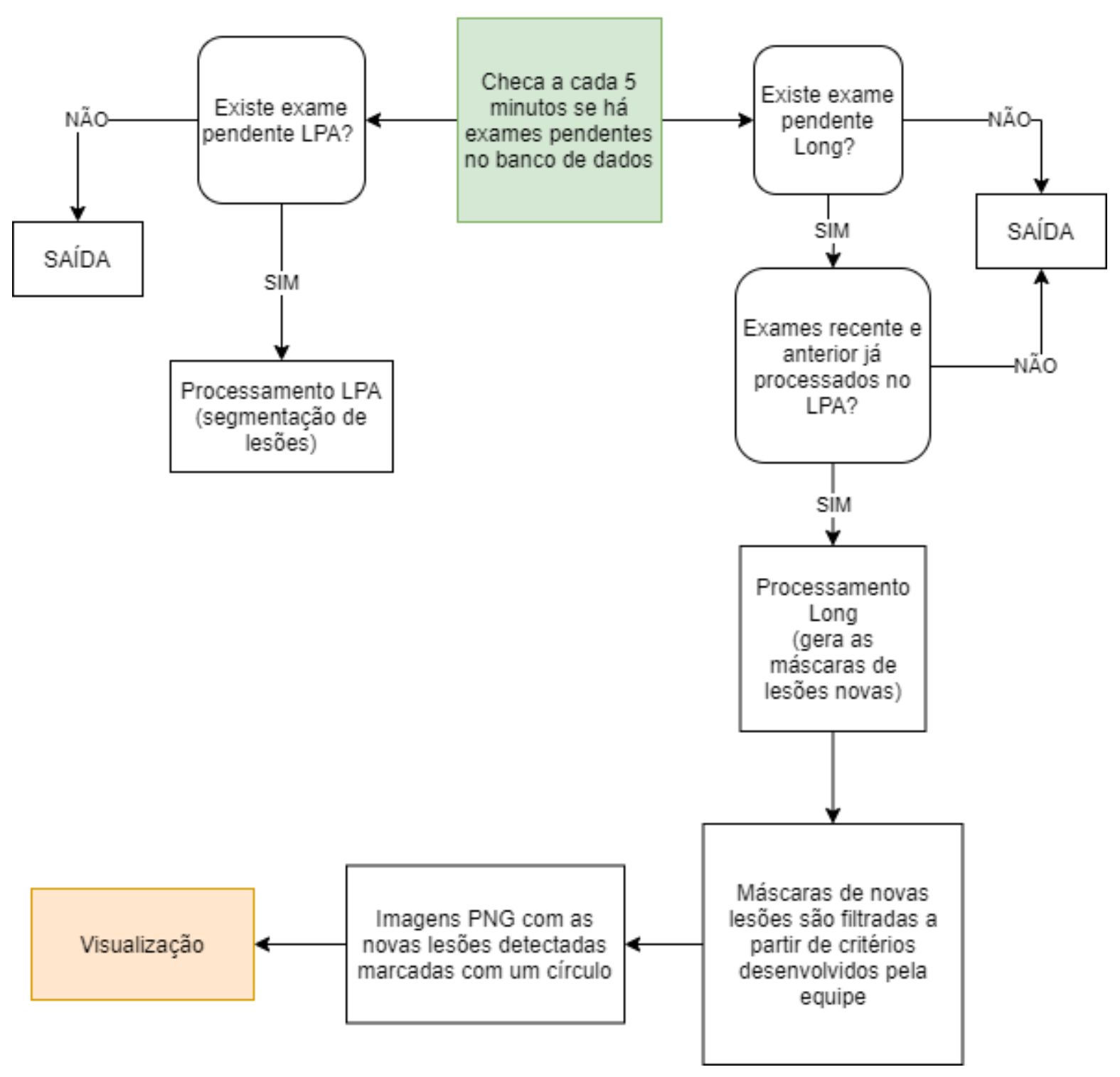

Figura 3: script python checa a cada 5 minutos no banco de dados se há exames para serem processados simultaneamente para LPA e Long. Caso positivo os exames são encaminhados para processamento, sendo uma checagem a mais feita antes do processamento Long para se certificar de que os exame anterior e recente já foram processados pelo LPA. 


\section{VISUALIZAÇÃO}

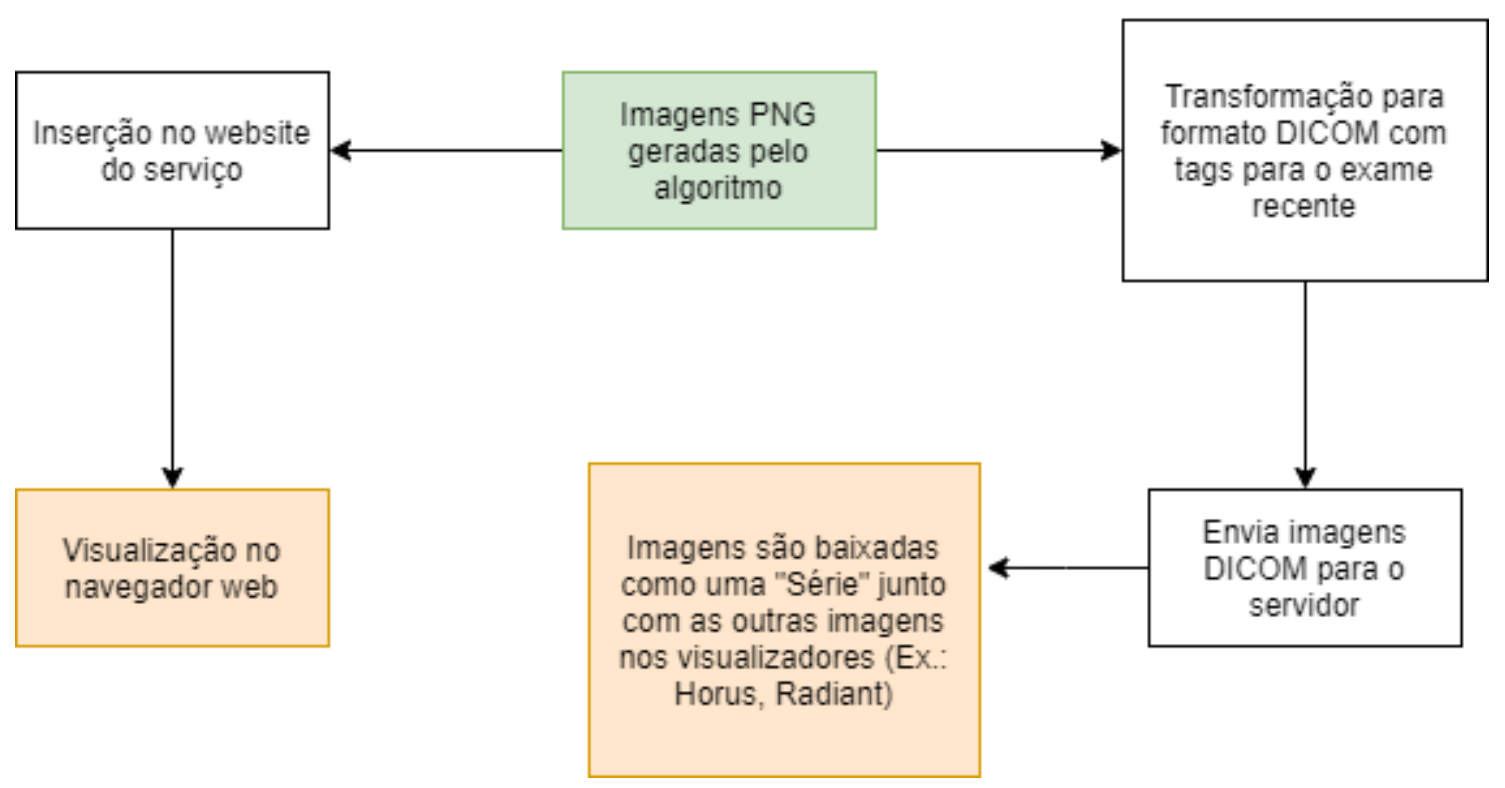

Figura 4: após o processamento Long as imagens PNG geradas são armazenadas no servidor e podem ser visibilizadas de duas formas: navegador web a partir de um site desenvolvido pela equipe que tem acesso às imagens e através do próprio visualizador de imagens DICOM usado para laudos médicos. Para possibilitar esta última forma de visualização desenvolvemos outro script que gera imagens DICOM a partir das imagens PNG com as tags específicas para possibilitar que sejam agrupadas juntamente com as imagens estruturais, lembrando que são adicionadas no estudo recente.

Visando cumprir o primeiro objetivo específico deste trabalho, um script na linguagem Python foi desenvolvido para verificar a cada 5 minutos a existência de novos exames de ressonância magnética no servidor DICOM do serviço de radiologia do HCFMRP. Caso seja detectado um novo exame o algoritmo faz a checagem se o registro do paciente está incluso na lista de pacientes com EM do HCFMRP armazenada no nosso banco de dados, se positivo o exame é extraído da base de dados e armazenado de forma separada, ainda em formato DICOM. Deve ser mencionado que a lista de pacientes está armazenada em um banco de dados 
SQL e pode ser facilmente atualizada pelo neuro-radiologista responsável através de um website desenvolvido por nós. Uma segunda fase deste primeiro objetivo é a verificação da existência de exames anteriores do paciente por um algoritmo, também implementado em Python, que filtra os exames de ressonância magnética e as sequências necessárias ao processamento, como vai ser visto logo a seguir. Se comprovada a existência de um exame anterior com as condições específicas requeridas para o processamento, o exame é disponibilizado para a segunda etapa da ferramenta implementada, o Processamento (Figura 3). 


\section{Resultados}

A nossa ferramenta procura uma máxima automatização do problema mencionado, isto

é, que não seja necessário nenhum passo de processamento por parte de qualquer profissional, sendo os exames feitos na máquina de ressonância automaticamente selecionados, processados e os resultados sobre novas lesões entregues juntamente com as imagens do exame ao médico radiologista no momento da elaboração do laudo. Um outro objetivo geral do nosso trabalho é a aplicação de ferramentas de software na prática clínica diária do radiologista, não unicamente para a visualização senão para a quantificação da informação contida nas imagens.

Deve-se destacar a importância da sensibilidade da ferramenta, sendo fundamental que seja a mais alta possível para obter o maior valor preditivo negativo possível. Temos a hipótese que o auxílio do processamento automático das lesões traga mais segurança ao médico radiologista nesta tarefa complicada e tediosa.

Dentre as imagens de RM usadas na prática clínica no acompanhamento dos pacientes de EM, a imagem FLAIR (do inglês, Fluid Attenuated Inversion Recovery) destaca-se pela capacidade de diferenciar placas de desmielinização da substância branca normal (8). 0 anterior pode ser verificado em imagens de um dos pacientes aqui avaliados (Figura 5). Adicionalmente, existe uma ferramenta disponível livremente online (https://www.appliedstatistics.de/Ist.html) que a partir da comparação de duas imagens FLAIR do mesmo indivíduo gera um mapa de probabilidades de distribuição de novas lesões (9). Por ambos os motivos, a aquisição FLAIR foi escolhida para ser utilizada na segmentação de lesões . 


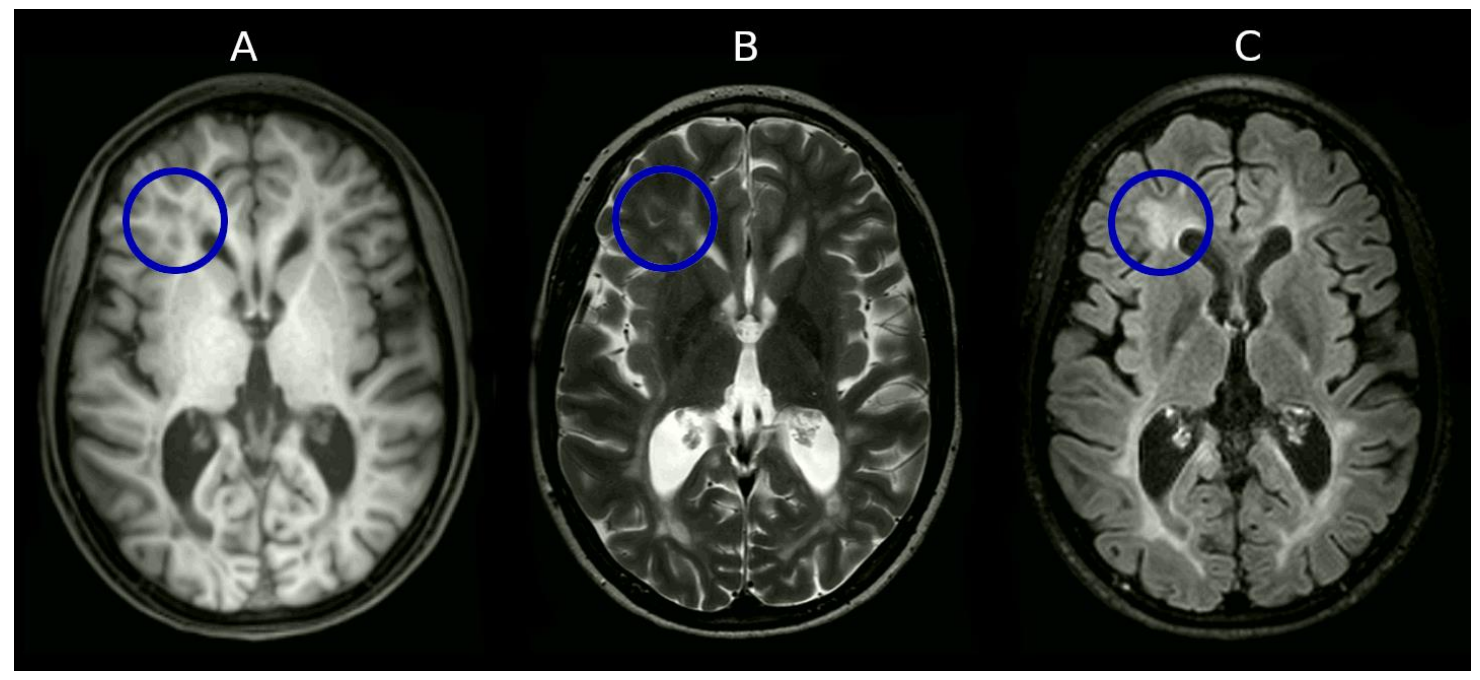

Figura 5: Exemplo de diferentes contrastes de imagens do encéfalo de um paciente de EM avaliado neste trabalho: a) T1, b) T2, c) FLAIR. Note-se a relativa facilidade de identificação de lesões na imagem FLAIR, salientadas com círculos azuis em todas as imagens.

Na primeira fase da etapa de Processamento usamos as imagens da sequência FLAIR, disponibilizadas na etapa anterior e obtidas no equipamento de RM mencionado com as seguintes características de aquisição: Sequência FLAIR 3D, Tempo de Repetição = $5000 \mathrm{~ms}$, Tempo ao Eco= 331,3 ms, Número de fatias= 169, Matriz de aquisição $=0 \backslash 240 \backslash 240 \backslash 0$. Nas duas imagens consecutivas do paciente usamos a função LPA na ferramenta LST (https://www.applied-statistics.de/Ist.html) desenvolvida no software Matlab e que adicionalmente usa a biblioteca SPM8 (https://www.fil.ion.ucl.ac.uk/spm/software/spm8/) desenvolvida também neste software. Essa função faz a segmentação das lesões em ambos os exames gerando um mapa de distribuição de probabilidades de lesões para ambos individualmente no espaço de ambas as imagens FLAIR (Figura 6). Adicionalmente, essa função compara os dois exames gerando um único mapa com a distribuição da evolução das lesões no espaço da segunda (ou posterior) imagem FLAIR (Figura 6), sendo identificadas regiões com aumento, diminuição ou estáveis com base em valores números discreto de pixels. 


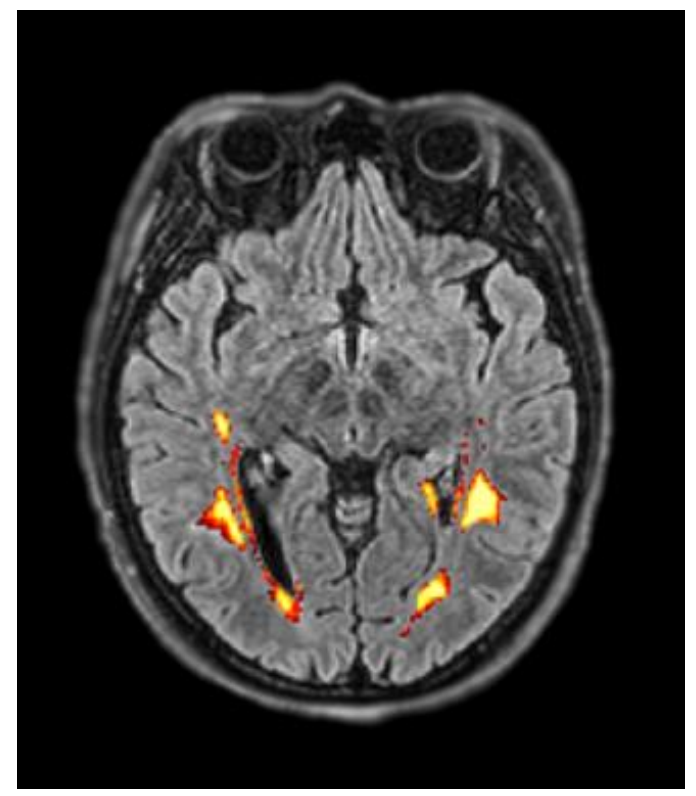

Figura 6: Exemplo do uso da função LPA da ferramenta LST (https://www.appliedstatistics.de/lst.html) em um paciente de EM avaliado neste trabalho: as regiões com alta probabilidade de serem lesões relacionadas a esclerose múltipla estão graduadas em um "heatmap".

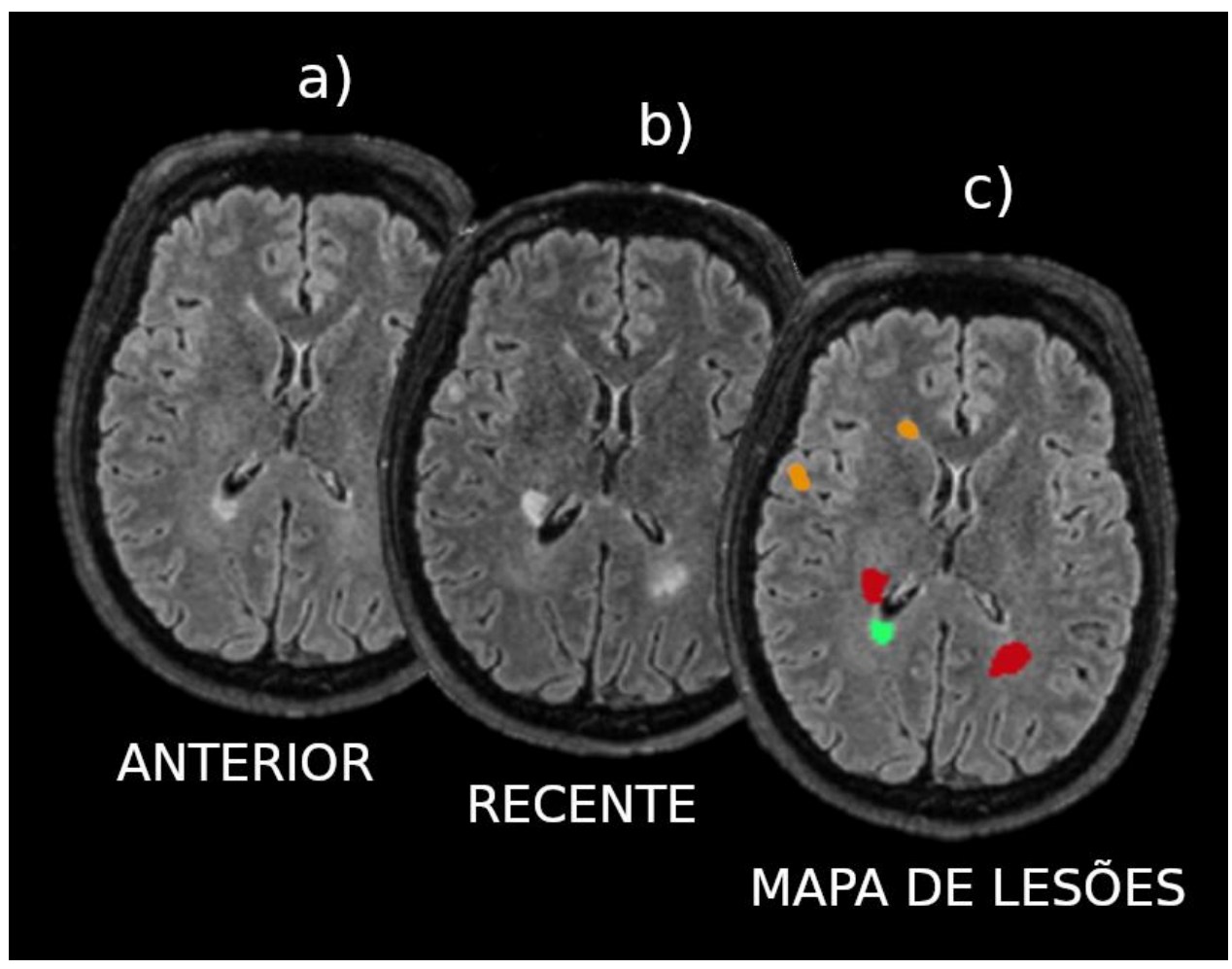


Figura 7: Exemplo do uso da função Long da ferramenta LST (https://www.appliedstatistics.de/Ist.html) em um paciente de EM avaliado neste trabalho: a) FLAIR inicial (anterior) co-registrado com o b) FLAIR posterior (recente), c) Mapa com a distribuição da evolução das lesões. Note-se no mapa são identificadas regiões de lesões com: Aumento (vermelho), Diminuição (verde) e Estáveis (laranja).

Após a geração do mapa de evolução das lesões, na segunda fase da etapa de Processamento aplicamos um algoritmo desenvolvido por nós usando Matlab para a clusterização e identificação das novas lesões. Nesse algoritmo primeiramente agrupamos ou clusterizamos as zonas com valores determinados empiricamente no mapa de evolução das lesões considerando uma conectividade mínima entre vizinhos, valor obtido empiricamente para evitar pequenas agrupações de voxels isolados. Como saída do nosso código são geradas as coordenadas dos voxels de cada nova lesão individual no espaço da segunda imagem FLAIR, assim como a posição espacial do centróide de cada lesão. Ainda neste código, ambas as informações são usadas para gerar para cada lesão nova arquivos no formato de imagem PNG de cortes axiais centrados na lesão, contendo as imagens FLAIR e uma elipse azul em volta de cada lesão, como mostra na figura 8. As dimensões da elipse desenhada leva em consideração o número de voxels clusterizados em cada nova lesão. Por último uma imagem PNG que resume os resultados é criado contendo as informações do número e volume de lesões nos dois exames comparados. 


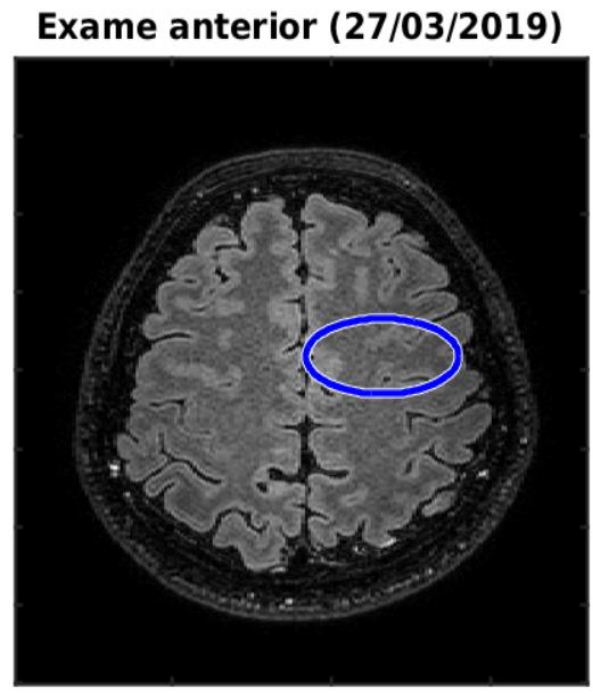

$\begin{array}{lcc}\text { Exame } & \text { Anterior } & \text { Recente } \\ \text { - } & & \\ \text { Data } & 22 / 06 / 2018 & 23 / 01 / 2020 \\ \text { Nro. de lesões } & 21 & \end{array}$

Vol. de lesões (ml) 4.24
Exame atual (28/11/2019)

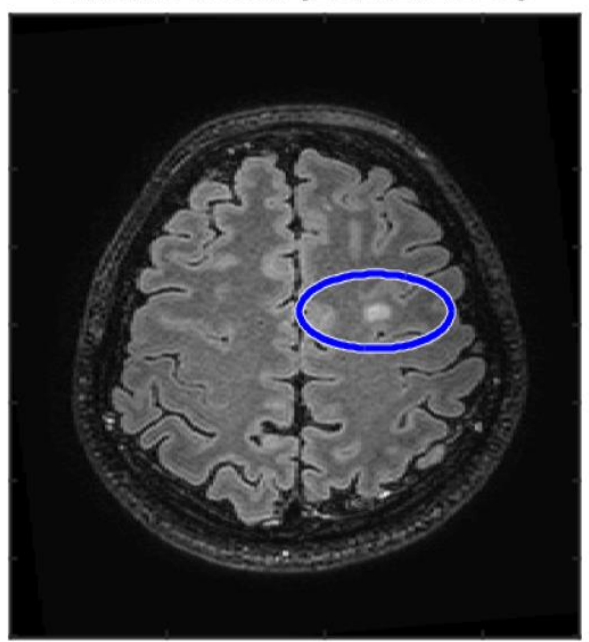

4.4

Figura 8: Exemplo de identificação de nova lesão (elipse azul) a partir da comparação de uma imagem FLAIR inicial (esquerda, exame anterior) e uma posterior (direita, exame atual). A imagem exibida representa um exemplo de arquivo PNG criado. Abaixo é mostrada a imagem com as informações do número e volume de lesões detectadas em cada exame.

A etapa de Visualização inicia-se com a conversão das imagens geradas no formato PNG para o formato DICOM utilizando as tags da própria imagem FLAIR do paciente visando aproveitar incorporar a maior quantidade de informações disponíveis sobre o paciente, entre elas: Nome, Registro, Identificação do exame, etc. Ainda são gerados registros DICOM novos específicos para cada imagem alterando os campos: Series/nstanceUID e SOPInstanceUID. Essa conversão é feita usando um algoritmo escrito na linguagem python que lê uma imagem do exame recente e gera novas tags. Na segunda fase da etapa de Visualização e com o uso de de outro algoritmo na linguagem python, as novas imagens DICOM geradas são transferidas para o 
servidor DICOM do serviço de Radiologia e passam a formar parte do estudo do paciente como uma Série. Esta nova série gerada pode ser baixada pelo médico radiologista juntamente com as outras imagens do exame em qualquer visualizador de imagens (Horus, Radiant, etc). $\mathrm{O}$ radiologista pode visualizar individualmente cada nova lesão detectada e/ou o relatório final incorporado. Como uma segunda alternativa de visualização e apresentação de resultados foi também desenvolvido um website com acesso interno a usuários cadastrados no HCFMRP para visualização dos exames processados e atualização da lista de paciente com EM, anteriormente mencionada. O website foi desenvolvido utilizando as linguagens python, html, javascript e react.

Nosso pipeline é totalmente automatizado, não exigindo nenhuma tarefa por parte dos técnicos na máquina de ressonância magnética ou no Centro de Imagens do HCRP, nem por parte do médico radiologista, que já recebe as imagens processadas no momento que vai elaborar o laudo. O pipeline envolveu o uso de diversas ferramentas de programação: servidor de banco de dados MySQL, linguagens de programação Python, Javascript, React e Matlab.

Para avaliar a sensibilidade, especificidade e acurácia da ferramenta proposta consideramos como padrão ouro para a existência de novas lesões os laudos médicos dos 99 exames mencionados e utilizamos os dados colectados pelo website desenvolvido.

A sensibilidade (S) foi definida como:

$\mathrm{S}=\mathrm{TP} /(\mathrm{TP}+\mathrm{FN})=0.95$

Onde, TP: Representa o número de verdadeiros positivos, FN: Representa o número de falsos negativos,.

No caso da especificidade (E) foi definida como:

$E=T N /(T N+F P)=0.31$

Onde, TN: Representa o número de verdadeiros negativos e FP: Representa o número de falsos positivos.

No caso da precisão $(P)$ foi definida como: 


$$
P=T P /(T P+F P)=0.96
$$

No caso da acurácia (A) foi definida como:

$A=(T P+T N) /(T P+F P+F N+T N)=0.46$

Valores: $\mathrm{TN}=22 ; \mathrm{FP}=1 ; \mathrm{FN}=52 ; \mathrm{TP}=24$ 


\section{Discussão}

Foram processados automaticamente, com o uso do pipeline proposto e até dezembro de 2019, 99 estudos de RM correspondentes a 99 pacientes com EM. O algoritmo detecta automaticamente a realização de exames de pacientes previamente inseridos na lista de pacientes com o diagnóstico, a partir da gravação do estudo no servidor DICOM do CCIFM.

A partir da realização do exame, é detectada a presença de sequência volumétrica FLAIR VISTA no estudo. A sequência volumétrica FLAIR do último exame é corregistrada com a volumétrica FLAIR do exame anterior, ambas são segmentadas com detecção automática de volume e número de lesões visíveis em T2. O algoritmo gera diversos pares de imagens, assinalando com um círculo aquelas consideradas novas lesões. Estas imagens, exemplificadas na figura 9 é informação nova incorporada aos estudos de imagem, sendo enviadas para o servidor DICOM e aparecendo como uma nova sequência do exame. Estas imagens ficam a disposição do médico que vai emitir o laudo para que seja conferida a efetividade da detecção. Estes resultados disponíveis no sistema foram comparados por dois médicos seniors, com treinamento em neuroimagem (Antonio Carlos dos Santos e Alessandro Spagnol) acrescidos de um médico residente de terceiro ano ou um médico de complementação especializada de neurorradiologia. Todos os casos foram verificados, imagem a imagem, para a detecção de falsos positivos e falsos negativos. 


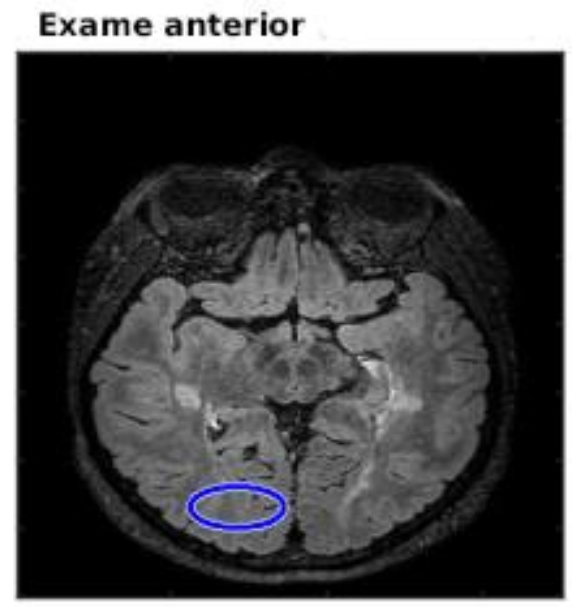

\section{Exame anterior}

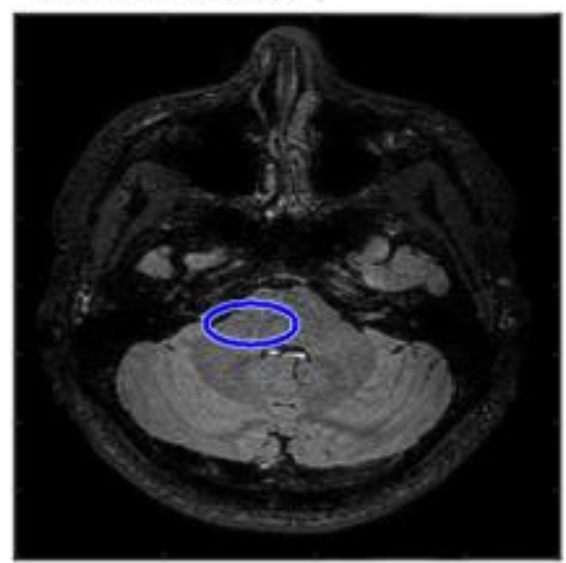

\section{Exame anterior}

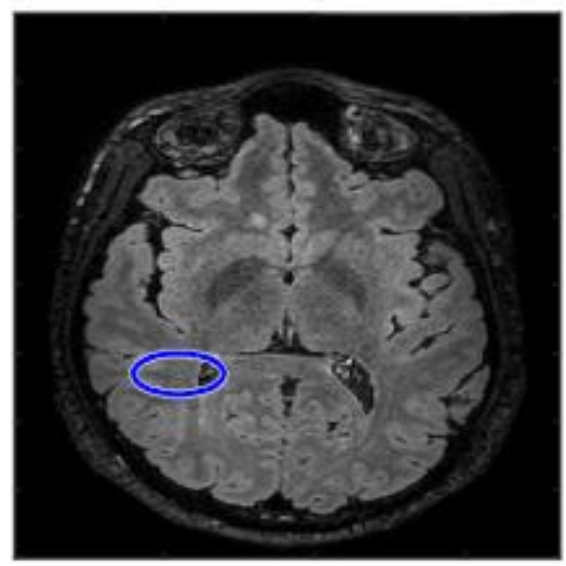

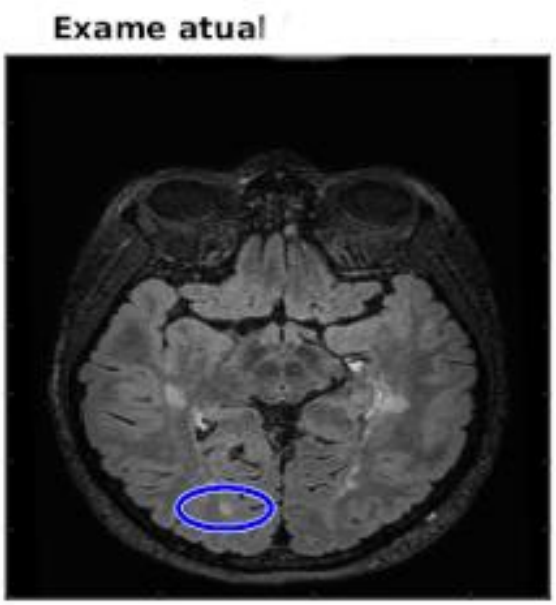

Exame atual

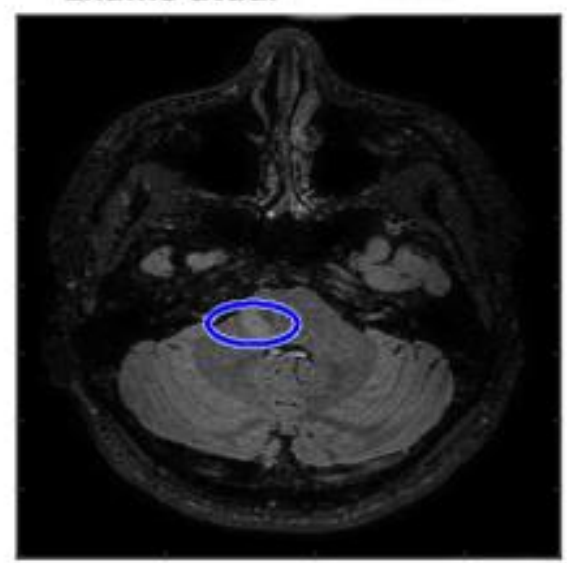

Exame atual

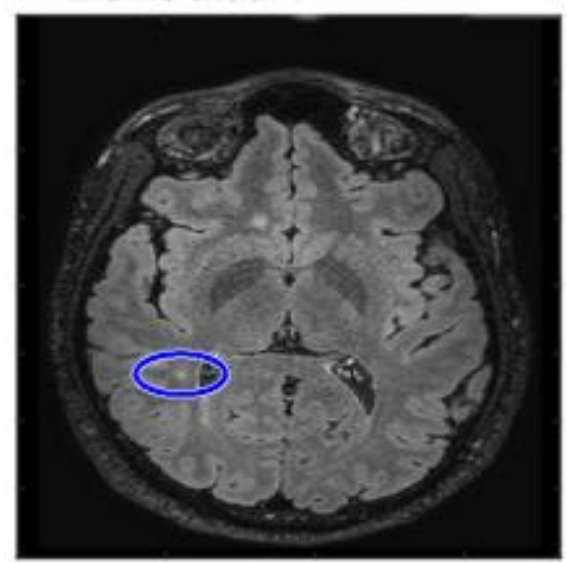

Figura 9: Exemplos de detecção de lesão nova pelo pipeline aqui proposto em diferentes pacientes com EM a partir das imagens FLAIR: Exame anterior (esquerda), Exame posterior (direita). A lesão nova em cada caso é indicada por uma elipse azul. Cada imagem contempla somente uma lesão nova marcada, sendo geradas pares de imagens para cada nova lesão. 
Foi observada uma sensibilidade de $95 \%$ e especificidade foi de $31 \%$, valor que pode ser considerado baixo, assim como acurácia que foi de $46 \%$. Obtivemos somente um falso negativo entre os 99 exames avaliados, com uma única lesão nova não detectada pela nossa metodologia (Figura 10).

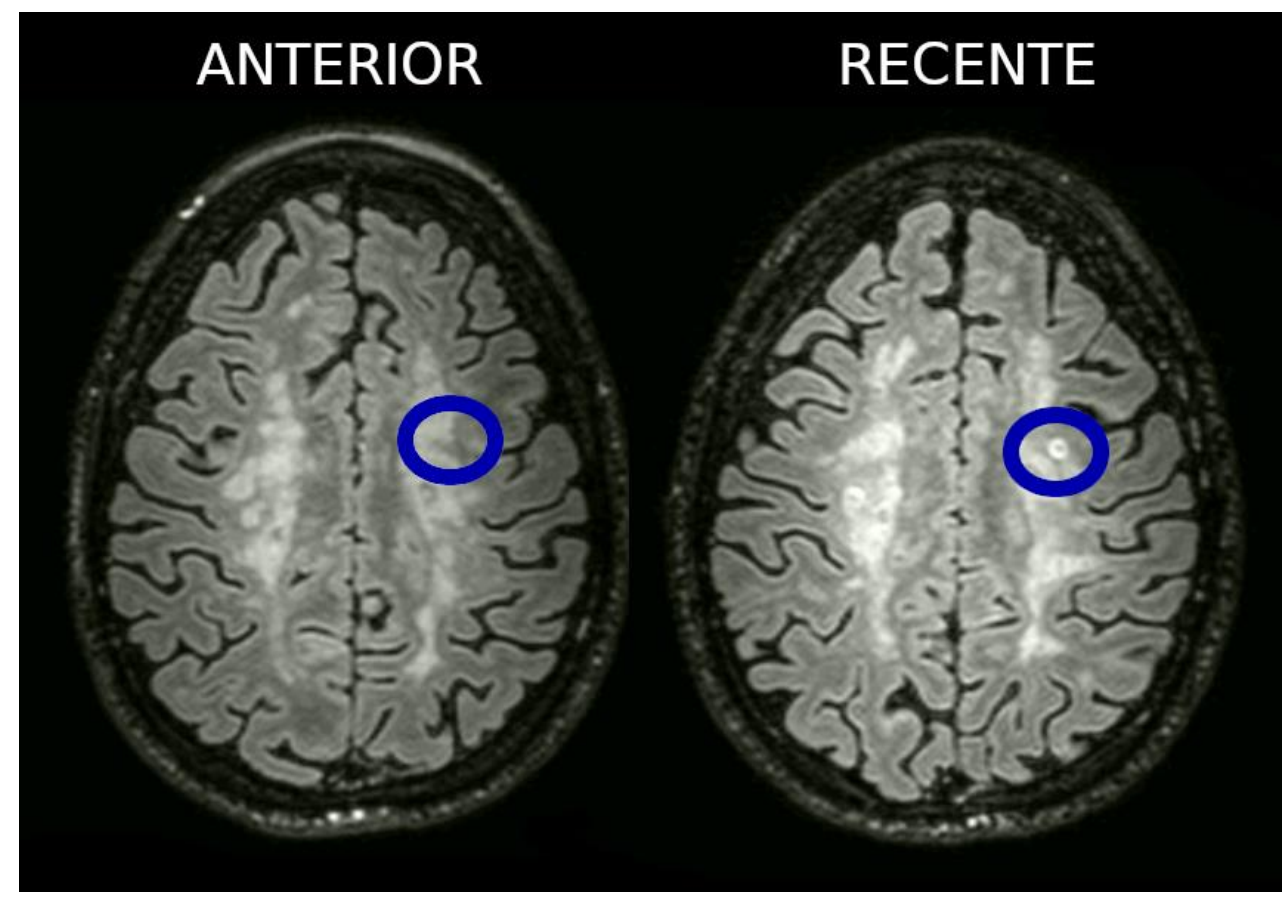

Figura 10: Imagens FLAIR de paciente considerado sem lesão nova pelo pipeline aqui proposto, porém com lesão nova no laudo médico no centro semioval esquerdo: Exame anterior (esquerda), Exame posterior (direita). Uma das lesões apontada no laudo é indicada com com um círculo azul.

No desenvolvimento do trabalho focamos em obter uma excelente sensibilidade, mesmo com prejuízo da especificidade, pois o intuito inicial não foi substituir o especialista médico, mas sim auxiliá-lo e evitar que um paciente ficasse sem uma nova lesão verdadeira detectada. Além da elevada sensibilidade, pequenas lesões novas, inferiores a $4 \mathrm{~mm}$, ou com um discreto aumento no contraste na região da lesão nova foram detectadas pelas nossa ferramenta (Figura 11). Estes achados são de difícil detecção pelo radiologista, especialmente 
em casos com abundância de lesões, e trazem um aumento de confiança no uso do pipeline proposto.

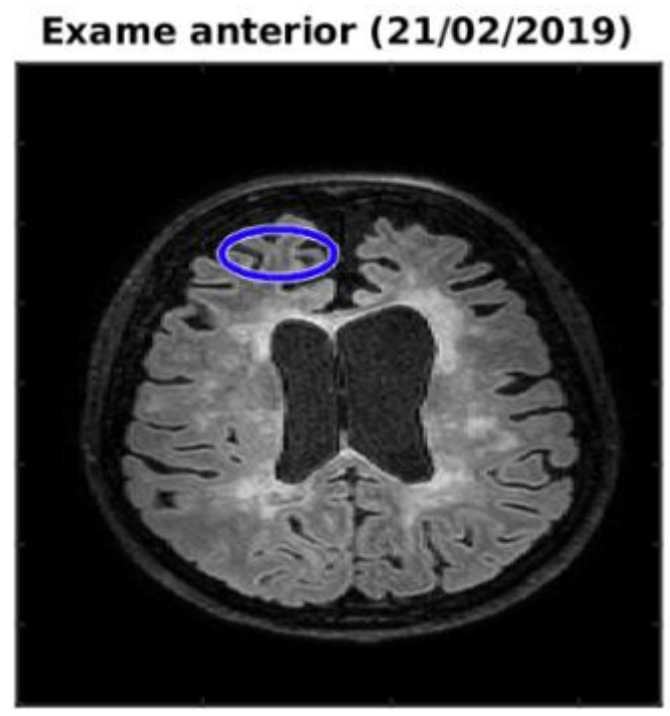

\section{Exame atual (27/11/2019)}

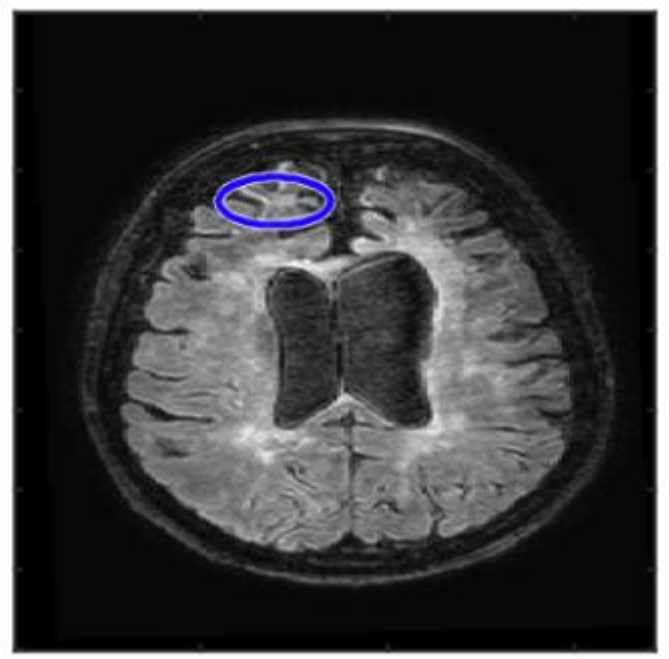

Figura 11: Imagens FLAIR de pacientes, registro individual indicado em cada caso, contendo exemplos de lesão nova muito pequena detectada pelo pipeline aqui proposto, e indicada por uma elipse azul.

Como mencionado, a especificidade foi baixa, aparecendo um alto número de falsos positivos. Felizmente encontramos certa regularidade dentre a maioria desses falsos positivos, podendo citar: aumentos discretos nas dimensões de lesões preexistentes (Figura 12), região do plexo coróide (Figura 13), lesões periventriculares (Figura 14). O aumento de tamanho discreto possui certa subjetividade em ser considerado em alguns casos como uma nova lesão dependendo da proximidade da lesão preexistente. A detecção de lesão nova na região do plexo coróide e em lesões periventriculares depende da orientação cefálica do paciente no momento da aquisição da imagem e o efeito do posterior coregistro de ambos os exames. Isto é, se o paciente ficou exatamente na mesma localização em ambos os exames ou o co-registro foi perfeito, uma estrutura com a mesma dimensão identificada como lesão, mesmo erroneamente, não será considerada lesão nova. 

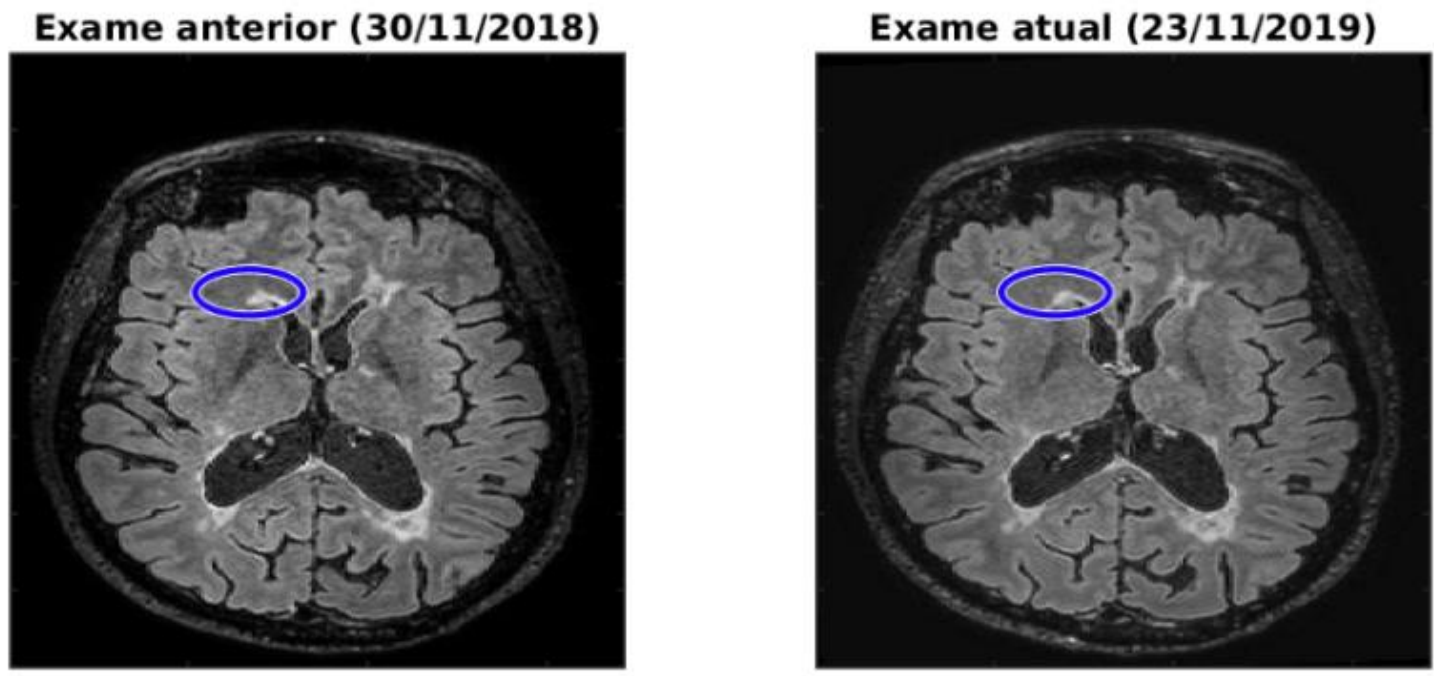

Figura 12: Imagens FLAIR de pacientes contendo exemplos de falsos positivos de lesão nova (elipse azul) detectada pelo pipeline aqui proposto, a partir de um aumento discreto de lesões preexistentes. À esquerda o exame anterior e o posterior à direita.
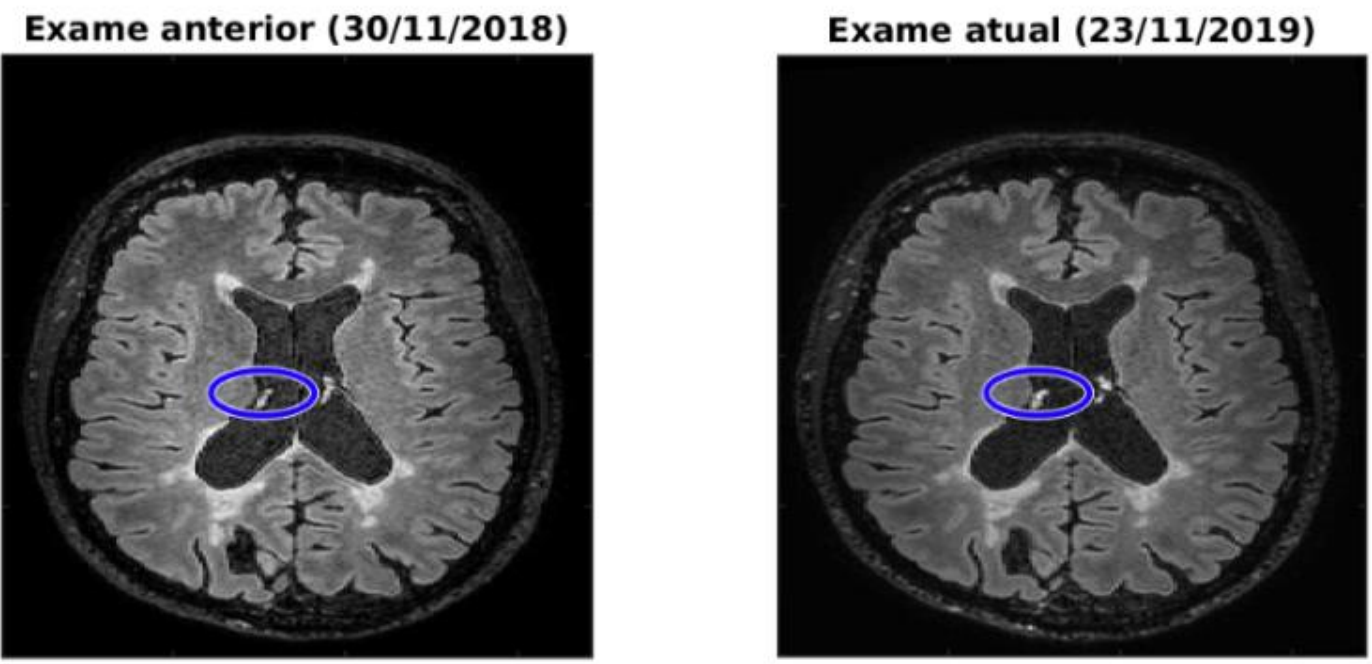

Figura 13: Imagens FLAIR de pacientes contendo exemplos de falsos positivos de lesão nova (elipse azul) detectada pelo pipeline aqui proposto na região plexo coróide. À esquerda o exame anterior e o posterior à direita. 
Exame anterior (30/11/2017)

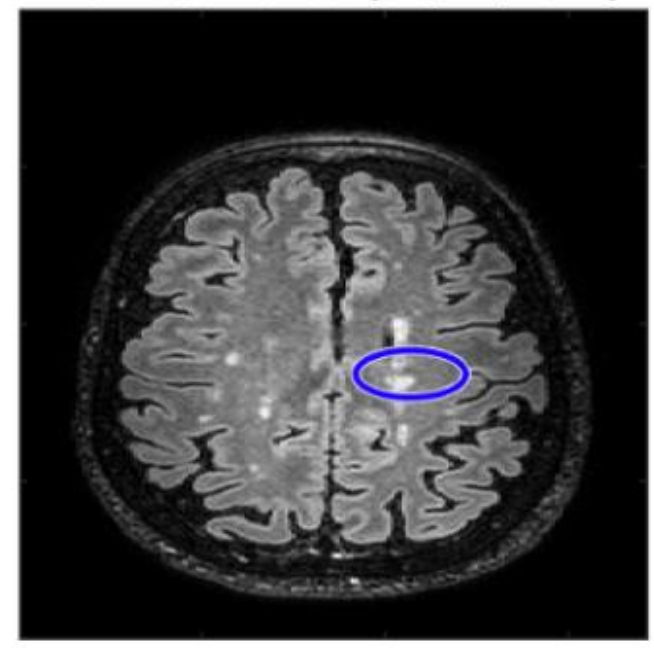

Exame atual (22/11/2019)

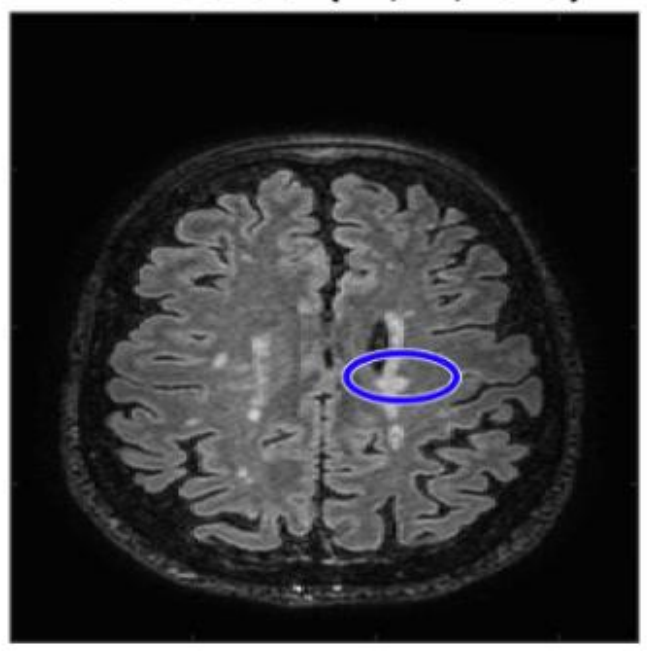

Figura 14: Imagens FLAIR de pacientes contendo exemplos de falsos positivos de lesão nova (elipse azul) detectada pelo pipeline aqui proposto em regiões com lesões periventriculares preexistentes. À esquerda o exame anterior e o posterior à direita.

Na literatura pesquisada encontramos outros métodos de detecção de novas lesões. Até o momento, não achamos nenhuma ferramenta que disponibilizasse de forma fácil, automatizada e direta os resultados da comparação dos exames para uso na prática clínica diária dos radiologistas.

A ferramenta desenvolvida já está em aplicação na prática diária dos neurorradiologistas do HCFMRP, que relatam de forma qualitativa um aumento significativo na detecção de novas lesões e maior segurança na hora de avaliar as imagens do exame. Os falsos positivos detectados até o momento, embora frequentes, são facilmente descartados pelo radiologista. 


\section{Conclusões e trabalhos futuros}

O objetivo primário deste trabalho de desenvolvimento de uma ferramenta de auxílio ao médico radiologista para a avaliação de dois exames consecutivos de imagens por RM de pacientes com Esclerose Múltipla visando a detecção automática de novas lesões foi cumprido, criando-se uma ferramenta de alta sensibilidade, superior a 95 \%. Foi conseguida a aplicação na rotina clínica da ferramenta desenvolvida com mais de 99 exames de pacientes avaliados até dezembro de 2019 e estando atualmente em execução automática no serviço de radiologia do HC-FMRP, tendo um retorno altamente positivo dos médicos radiologistas do serviço.

O pipeline implementado foi formado por três principais etapas (Seleção, Processamento e Visualização).

Há algumas limitações da ferramenta implementada, principalmente relacionadas com o procedimento de detecção de lesões, desenvolvido por (9). Este procedimento é limitado a imagens provenientes de um aquisição tipo $F L A I R$ e resulta em falsos positivos em regiões anatômicas com hipersinal neste tipo de aquisições como por exemplo o plexo coróide. Na nossa experiência, a sequência GM only parece ser mais sensível para avaliar a presença de lesões desmielinizantes de Esclerose Múltipla, por possuir maior contraste que a sequência FLAIR. Ainda os critérios aqui definidos para a identificação de lesões novas podem ser aprimorados visando a redução de falsos positivos.

Nossa avaliação quantitativa da ferramenta foi limitada à análise de cada paciente e não de cada lesão individual, assim como nosso padrão ouro, o laudo médico, não foi revisto após as indicações da ferramenta para uma segunda avaliação e discussão individual mais aprofundada. Limitações adicionais da nossa ferramenta incluem erros diversos que eventualmente ocorrem durante o processamento e que estão sendo corrigidos, entre eles podemos citar: falhas na seleção das imagens, eventuais falhas no LST e a necessidade eventual de reiniciar o sistema devido a falhas no sistema operacional.

Atualmente, estamos desenvolvendo nossa própria ferramenta de detecção e segmentação de lesões de Esclerose Múltipla utilizando técnicas de Deep Learning. Com este novo método espera-se uma maior acurácia, reduzindo o número de falsos positivos, seu 
tempo de processamento é cerca de 100 vezes menor do que o método que utilizamos hoje, e ainda pode ser utilizado em imagens provenientes da sequência $G M$ only. 


\section{Referências:}

1. Ropper, A.H. and Brown, R.H. (2005) Cerebrovascular diseases. In: Adams and Victor's Principles of Neurology, 8th Edition, McGraw-Hill Co., Inc., New York, 34, 682-684.

2. [Referências: tese de livre docente de Antônio Carlos e de doutorado da Doralina Guimarães Brum Souza]

3. Tintore, M., Rovira, À., Río, J., Otero-Romero, S., Arrambide, G., Tur, C., ... Montalban, X. (2015). Defining high, medium and low impact prognostic factors for developing multiple sclerosis. Brain, 138(7), 1863-1874. https://doi.org/10.1093/brain/awv105

4. Choy, G., Khalilzadeh, O., Michalski, M., Do, S., Samir, A. E., Pianykh, O. S., ... Dreyer, K. J. (2018). Current Applications and Future Impact of Machine Learning in Radiology. Radiology, 288(2), 318-328. https://doi.org/10.1148/radiol.2018171820

5. Salem, M., Cabezas, M., Valverde, S., Pareto, D., Oliver, A., Salvi, J., Rovira, À., \& Lladó, X. (2018). A supervised framework with intensity subtraction and deformation field features for the detection of new T2-w lesions in multiple sclerosis. Neurolmage: Clinical, 17, 607-615. https://doi.org/10.1016/j.nicl.2017.11.015

6. Cabezas, M., Corral, J. F., Oliver, A., Díez, Y., Tintoré, M., Auger, C., ... Rovira, À. (2016). Improved Automatic Detection of New T2 Lesions in Multiple Sclerosis Using Deformation Fields. American Journal of Neuroradiology, 37(10), 1816-1823. https://doi.org/10.3174/ajnr.a4829

7. Galletto Pregliasco, A., Collin, A., Guéguen, A., Metten, M. A., Aboab, J., Deschamps, R., Gout, O., Duron, L., Sadik, J. C., Savatovsky, J., \& Lecler, A. (2018). Improved Detection of New MS Lesions during Follow-Up Using an Automated MR Coregistration-Fusion Method. American Journal of Neuroradiology, 39(7), 1226-1232. https://doi.org/10.3174/ajnr.a5690

8. Pretorius, P. ., \& Quaghebeur, G. (2003). The Role of MRI in the Diagnosis of MS. Clinical Radiology, 58(6), 434-448. https://doi.org/10.1016/s0009-9260(03)00089-8

9. Lesions were segmented by the lesion prediction algorithm (Schmidt, 2017, Chapter 6.1) as implemented in the LST toolbox version 3.0.0 (www.statistical-modelling.de/lst.html) for SPM. 
